\title{
INDIGENOUS RECOMBINANT STREPTOKINASE VS NATURAL STREPTOKINASE IN ACUTE MYOCARDIAL INFARCTION PATIENTS: PHASE III MULTICENTRIC RANDOMIZED DOUBLE BLIND TRIAL
}

S. K. DIWEDI, J. S. HIREMATH ${ }^{1}$, P. G. KERKAR ${ }^{2}$, KRISHNA N. REDDY ${ }^{3}$, C. N. MANJUNATH ${ }^{4}$, S. S. RAMESH ${ }^{5}$, S. PRABHAVATI ${ }^{4}$, M. DHOBE ${ }^{1}$, KAVITA SINGH $^{6}$, P. BHUSARI ${ }^{6}$, RAMAN RAO 6

\section{ABSTRACT}

BACKGROUND: Streptokinase is the most widely used thrombolytic agent and can now be made using recombinant DNA technology. The present trial was initiated to assess an indigenous recombinant streptokinase (Shankinase, r-SK). AIM: To compare the efficacy and safety of indigenous recombinant streptokinase (Shankinase, $r-S K$ ) and natural streptokinase (Streptase, n-SK). SETTINGS AND DESIGN: Double blind, randomized, non-inferiority, multicentric, parallel study. MATERIALS AND METHODS: Patients of AMI < 6 hours of chest pain and $2 \mathrm{~mm}$ ST elevation in 2 contiguous chest leads $V_{1}-V_{6}$ or $1 \mathrm{~mm}$ in limb leads were randomized to receive 1.5 miu of either $r$-SK or n-SK. CK Peaking and decrease of $>50 \%$ ST segment were used to assess reperfusion. STATISTICAL ANALYSIS: Difference in the groups was assessed by chisquare or paired $t$ test as required. Probability value $<0.05$ was considered significant with $95 \%$ confidence interval. RESULTS: Overall 150 patients were recruited (96 r-SK group and 54 in $n$-SK group) and demographic and clinical profile of the groups was comparable. Reperfusion was seen in $68.2 \%$ (58) and $69.4 \%$ (34) patients in r-SK and n-SK groups respectively. Commonly seen adverse events were fever in 7 (8.5\%), hypotension in $3(3.6 \%)$, nausea in $2(2.4 \%)$ patients. Minor bleeding were seen in 4 $(4.8 \%)$ of patients. CONCLUSION: Indigenous recombinant Streptokinase (r-SK) is as efficacious as natural streptokinase (n-SK) in establishing reperfusion as assessed by non-invasive parameters with comparable side effect profile.

KEY WORDS: Streptokinase, Recombinant streptokinase, Myocardial infarction, Thrombolysis

\section{INTRODUCTION}

Over the last few years intravenous thrombolysis has become the standard

Department of Cardiology K.G.M.C. Hospital, Lucknow, ${ }^{1} \mathrm{CCU}$ Ruby Hall Clinic, Pune, ${ }^{2}$ Department of Cardiology KEM Hospital, Mumbai, ${ }^{3}$ CARE Hospital, Hyderabad, ${ }^{4}$ Department of Cardiology, Sri Jayadeva Institute of Cardiology, Bangalore, ${ }^{5}$ Heart Center, Bangalore, therapeutic approach for patients with acute myocardial infarction (AMI). Intervention with thrombolytic agents in acute myocardial infarction is an effective means of limiting

${ }^{6}$ Clinical Research Division, Shantha Biotechnics Ltd., Hyderabad, India

Correspondence:

Dr. Raman Rao, Head, Clinical Research Division, Serene Chambers, $3^{\text {rd }}$ Floor, Road No.7, Banjara Hills, Hyderabad 500 034, AP, India. E-mail: drraman@shanthabiotech.com 
myocardial damage. The reduction in short and long-term mortality rates caused by thrombolytics has been demonstrated in several large-scale clinical trials. ${ }^{[1-3]}$ Despite a slight difference in mortality found in the GUSTO trial, favoring tissue plasminogen activator (t-PA) when given as an accelerated infusion, the effects of natural streptokinase ( $n$ SK) on the outcome of the patients with AMI has been similar to other thrombolytic agents and it remains the agent of choice in India as it is cheaper. ${ }^{[4-6]}$ Though the greatest benefit occurs when streptokinase infusion is initiated early after onset of symptoms, late benefit has also been observed in patients treated even after $24 \mathrm{hrs}$ of onset of symptoms. ${ }^{[1,7]}$

Presently all available streptokinase for use in India, are naturally derived and expensive, while Shankinase is the first indigenously manufactured streptokinase obtained by means of recombinant-DNA technology. The recombinant-DNA process entails the culturing of $E$. coli carrying appropriate plasmid DNAs encoding for recombinant natural-type streptokinase followed by cell-lysis, refolding of streptokinase to their biologically active states, and their isolation at approximately $98 \%$ purity by chromatographic means.

This study was conducted at 6 centers, with the objective of comparing the effects of recombinant ( $r-S K$ ) and natural ( $n-S K$ ) streptokinase on coronary reperfusion in patients with acute myocardial infarction (AMI) and also the side effects.

\section{MATERIALS AND METHODS}

The present comparative, double blind, randomized, multicentric, parallel study between natural and recombinant Streptokinase was carried out in six hospitals across the country between January and June 2003. The medical division of Shantha Biotechnics, Hyderabad was responsible for coordinating and monitoring the study. An independent data monitoring safety board monitored all the events and serious adverse events regularly throughout the trial. The ethics committee at each hospital, and the Drug Controller General of India approved the protocol.

\section{Study patients}

Eligibility criteria: Patients with characteristic chest pain (ischemic origin) occurring for not more than 6 hours were considered for inclusion if there was $2 \mathrm{~mm}$ ST segment elevation in at least 2 contiguous chest leads or $1 \mathrm{~mm}$ elevation in standard limb leads. There was no gender restriction but patients below 65 years were enrolled.

Patients with previous or ongoing cerebrovascular disease, anticoagulant therapy, history of gastrointestinal hemorrhage or acute peptic ulcer, severe trauma, surgery, or invasive procedures in preceding 8 weeks, bleeding tendency, systemic BP ( $>180 / 110$ $\mathrm{mm}$ of $\mathrm{Hg}$ ), severe hepatic or renal failure, metastasizing malignancies and other terminal diseases, Streptokinase therapy within 5 days to 6 months, streptococcal disease within the previous 6 months, cardiogenic shock, allergy to Streptokinase or aspirin, proliferative diabetic retinopathy, infective endocarditis, and pregnancy or lactating were excluded.

\section{Study procedure}

Written informed consent was taken from the patients who were randomlized in 2:1 fashion (in favour of Shankinase r-SK) to receive 1.5 million units of recombinant Sreptokinase (Shankinase, Shantha Biotechnics Ltd., India) or natural Streptokinase (Streptase, HoechstRoussel, Germany) diluted in $100 \mathrm{ml}$ saline through a peripheral vein over a period of 30 $60 \mathrm{~min}$. Intravenous hydrocortisone $(100 \mathrm{mg}$ ) and oral aspirin $(325 \mathrm{mg}$ ) was administered to all patients. The decision of administration of concomitant medications was left to the physician.

\section{Objectives}

Primary objective: To establish reperfusion and compare the reperfusion rates in both the groups.

Secondary objective: To establish and compare the safety profile in each group.

\section{Outcomes: (Definitions of positive markers} of coronary artery patency)

Maximal CK activity within $12 \mathrm{hr}$ after the start of thrombolytic therapy was considered a positive marker of infarct related artery patency ${ }^{[8]}$ A positive ST marker was defined as a reduction in ST segment elevation of $\geq 50 \%$ within 90 mins after the start of thrombolysis. ${ }^{[8]}$ Pain reduction of more than $50 \%$ after 90 minutes of thrombolysis was also considered an indicator of reperfusion. ${ }^{[7]}$

\section{Determination of CK}

Venous blood samples were collected before administration of Streptokinase and then again at 90 mins, 6 hrs, $12 \mathrm{hrs}, 24 \mathrm{hrs}$ and 36 hours after infusion. CK activity was estimated by Sigma Diagnostics Creatinine Kinase Kit (Procedure No. 47-UV, Sigma Diagnostics, US)

\section{ECG assessment}

Standard 12 lead ECG was done immediate before and 90 minutes after initiation of Streptokinase infusion and was assessed and interpreted by two different observers to avoid bias in reporting. Lead with maximum ST segment elevation was identified and level of ST segment elevation was measured immediately prior to and 90 min after initiation of therapeutic therapy.

\section{Pain relief assessment}

The percentage decrease in pain was recorded at 90 minutes after initiation thrombolytic therapy on a scale of $0-10$ as perceived by the patient.

\section{Other investigations}

Serum samples were collected wherever possible for detecting anti-streptococcal antibodies. Pre discharge Echocardiogram was done to assess left ventricular function.

Liver function and renal function tests of all patients were carried before and after therapy. In case of bleeding episodes hemoglobin estimations were done to differentiate minor from major bleeds. Minor bleed was defined as bleeding not requiring transfusion and without hemodynamic compromise. Major haemorrhage was defined as bleeding requiring transfusion of blood or overt clinical bleeding (or documented intracranial or retroperitoneal hemorrhage) associated with a drop in hemoglobin of greater than $2 \mathrm{~g} / \mathrm{dl}$.

\section{Statistical analysis}

The sample size was determined as per regulatory requirements (schedule $Y$ of drugs and cosmetics act 1940 , where a study drug 
has to be evaluated in at least 100 subjects). The difference in both the groups was assessed by the chi-square and paired t test wherever required. A probability value $<0.05$ was considered to be significant with $95 \%$ confidence interval. The SAS version 8.2 (SAS Institute Inc, Cary, NC, US) software was used to carry out the statistical analysis.

\section{Randomization procedure}

Block randomization was done by using computer generated codes using the SAS software version 8.2 (SAS Institute Inc, Cary, NC, US).

\section{Random allocation procedures}

To preserve the blinding of the study, only a minimum number of personnel at the clinical research department where the code was generated had seen the random assignment list. Sealed emergency codes were available to the independent clinical monitor. These codes, which revealed the subject's drug group when opened, were only allowed to be opened during the study only if the choice of follow-up treatment depended on the subject's therapy assignment. If a subject's therapy assignment was unblinded, the department was notified immediately by telephone or fax. After the study the centers where the study was conducted returned all sealed and any opened codes.

\section{RESULTS}

\section{Clinical data}

Between January 2003 to June 2003, 150 were patients recruited in the study while 96 received recombinant 54 patients were administered natural streptokinase. As per protocol 85 out of 96 and 49 out of 54 patients were eligible for evaluation for efficacy while as per intention to treat all 150 were evaluated for safety. The organizational chart of the recruitment of the patients is represented in Figure 1. There was no significant difference in the demographic and clinical profile of patients in both the groups [Table 1]. Mean symptom-treatment interval was $3.6 \pm 2.5 \mathrm{hrs}$ and 3.8 $\pm 2.1 \mathrm{hrs}$ for Shankinase and Streptase respectively.

\section{Reperfusion}

CK peaking less than or at 12 hours was seen in $83.5 \%(71)$ and $89.8 \%$ (44) patients in r-SK and $\mathrm{n}$-SK groups respectively. The kinetics of

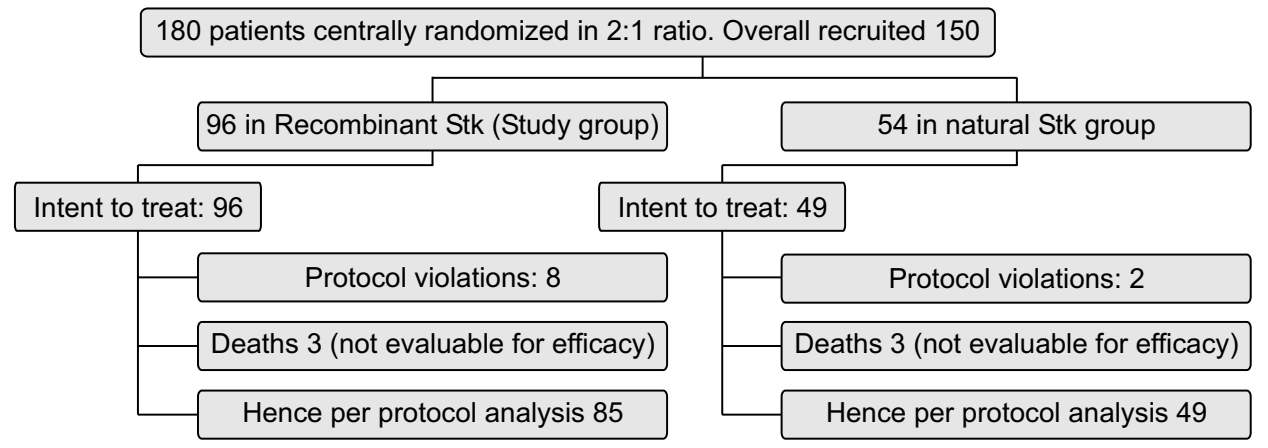

Figure 1: Patients and the recruitment scheme

Table 1: Demographic and clinical characteristics of patients

\begin{tabular}{llll}
\hline & $\begin{array}{l}\text { Recombinant } \\
\text { Streptokinase (\%) }\end{array}$ & $\begin{array}{l}\text { Natural } \\
\text { Streptokinase (\%) }\end{array}$ & 'P' value \\
& $52.0 \pm 10.3$ & $51.6 \pm 10.7$ & 0.822 \\
\hline Age (year, mean \pm SD) & $1: 6$ & 1.5 & NA \\
Sex (F: M) & $23(23.9)$ & $14(25.9)$ & 0.843 \\
Diabetes Mellitus & $25(26.0)$ & $14(25.9)$ & 0.929 \\
Hypertension & $14(14.6)$ & $8(14.8)$ & 0.989 \\
Previous Angina & $48(50.0)$ & $28(51.8)$ & 0.926 \\
Smoking & $5(5.2)$ & $1(1.8)$ & 0.303 \\
Previous MI & $7(7.3)$ & $4(7.4)$ & 0.971 \\
Obesity & $3.6 \pm 2.5$ (range & $3.8 \pm 2.1$ (range & 0.466 \\
Symptoms treatment & 50 minutes to 5.5 hrs) & 45 min to 5.3 hrs) & \\
interval (Hrs) & & & \\
Killips class & $85(88.5)$ & $45(83.3)$ & 0.435 \\
I & $7(7.3)$ & $7(13.0)$ & 0.435 \\
II & $2(2.1)$ & $2(3.7)$ & 0.435 \\
III & 0 & 0 & 0.435 \\
IV & & & \\
\hline
\end{tabular}

CK peaking at different intervals in both the groups is represented in Figure 2. ST segment resolution $\geq 50 \%$ was observed in $81.2 \%$ (69) and $79.6 \%$ (39) patients in $r-S K$ and $n-S K$ group respectively. Pain resolution in $94.1 \%$ (80) and $93.9 \%$ (46) of patients in r-SK and nSK group respectively. Reperfusion as assessed by the combination of CK peaking and ST resolution $\geq 50$ was seen in $68.2 \%$ (58) and $69.4 \%$ (34) in r-SK and n-SK groups respectively. When assessed by all three parameters reperfusion rates where $69.0 \%$ (59) and $65.3 \%$ (32) in r-SK and $n-S K$ respectively ( $P=$ N.S.) [Table 2].

Overall 47 serum samples were tested (27

from r-SK and 20 from n-SK group) for Antistreptococcal antibodies. As is evident from the Table 3 presence of anti-streptococcal

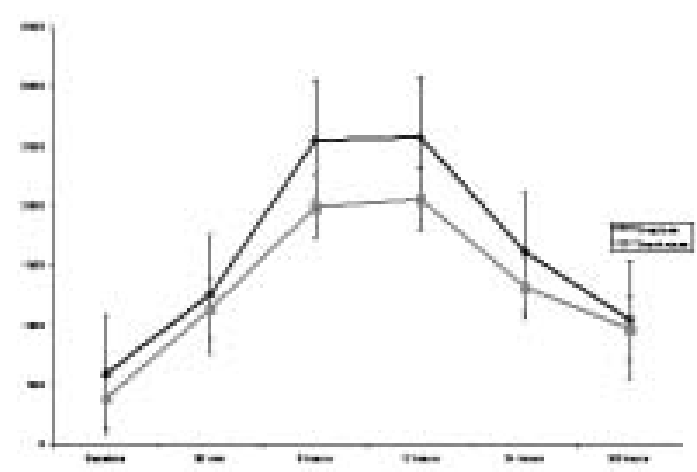

Figure 2: Mean CK values at different intervals in the two groups

Table 2: Efficacy parameters seen in both groups

\begin{tabular}{lccc}
\hline Efficacy parameters & \multicolumn{2}{c}{ Percent efficacy } & 'P' value \\
\cline { 2 - 3 } & $\begin{array}{c}\text { Recombinant } \\
\text { Streptokinase \% }(n)\end{array}$ & $\begin{array}{c}\text { Natural } \\
\text { Streptokinase \% (n) }\end{array}$ & \\
\hline CK Peaking $\leq 12$ hours & $83.5(71)$ & $89.8(44)$ & 0.293 \\
ST resolution $\geq 50 \%$ & $81.2(69)$ & $79.6(39)$ & 1.000 \\
Pain resolution & $94.1(80)$ & $93.9(46)$ & 1.000 \\
CK and ST resolution & $68.2(58)$ & $69.4(34)$ & 1.000 \\
CK, ST resolution and pain relief & $69.0(59)$ & $65.3(32)$ & 0.652 \\
\hline
\end{tabular}


Table 3: Concomitant medication in both the groups

\begin{tabular}{lcc}
\hline Concomitant Medication administered during study & $\begin{array}{c}\text { Recombinant } \\
\text { StreptokinaseN (\%) }\end{array}$ & $\begin{array}{c}\text { Natural } \\
\text { Streptokinase N (\%) }\end{array}$ \\
\hline Heparin & $30(31.2)$ & $17(31.4)$ \\
Beta blockers & $15(15.6)$ & $21(38.9)$ \\
ACE inhibitors & $19(19.8)$ & $23(42.6)$ \\
Angiotensin receptor blockers & $1(1.04)$ & $0(0)$ \\
Diuretics & $6(6.1)$ & $6(11.1)$ \\
Lipid lowering agents & $20(20.8)$ & $15(27.8)$ \\
Aspirin & $30(31.1)$ & $25(46.3)$ \\
Clopedogril & $26(27.1)$ & $17(31.5)$ \\
Morphine & $1(1.1)$ & $1(1.8)$
\end{tabular}

antibodies did not interfere with the reperfusion, in either of the groups.

The occurrence of reperfusion arrhythmias was observed in $25 \%$ of patients in both the groups. The most commonly seen arrhythmias were accelerated idoventricular rhythm (AIVR) $(38.8 \%)$, ventricular premature beats $(38.8 \%)$ and ventricular tachycardia (22.2\%)

\section{Adverse Events}

No significant differences were observed in the adverse event profile between the two groups although hypotension and fever were relatively common (Table 4). Hypotension in all the patients responded to inotropes and saline infusion after transient cessation of therapy.

\begin{tabular}{lcc} 
Table 4: Adverse events seen in both the groups \\
\hline Adverse events & \multicolumn{2}{c}{ Drug related } \\
\cline { 2 - 3 } & $\begin{array}{c}\text { Recombinant } \\
\text { Streptokinase } \\
N(\%)\end{array}$ & $\begin{array}{c}\text { Natural } \\
\text { Streptokinase } \\
N(\%)\end{array}$ \\
\hline Hypotension & $3(3.6)$ & $4(7.2)$ \\
Fever & $7(8.5)$ & $5(9.2)$ \\
Rigors & $3(3.6)$ & 0 \\
Nausea vomiting & $2(2.4)$ & $3(5.4)$ \\
Bleeding & & \\
Major & 0 & $1(1.8)$ \\
Minor & $4(4.8)$ & $3(5.4)$ \\
Angioedema & 0 & $1(1.8)$ \\
Anaphylaxis & 0 & $1(1.8)$ \\
\hline
\end{tabular}

managed conservatively.

\section{DISCUSSION}

Reperfusion in the present study which was the primary end point was asseseed using a combination of enzyme (Creatine Kinase) peaking and ST segment resolution was observed in $68.2 \%$ (58) in the $\mathrm{r}$-SK and in $69.4 \%$ (34) patients in n-SK group whereas, rates reported in literature by the present criteria vary from 59 to $82 \% .^{[1,4-6,9]} \mathrm{A}$ randomized comparative study was carried out in over 200 patients where a reperfusion rate of 67.1 and 70.7 was observed with recombinant and natural streptokinase respectively. ${ }^{[10]} \mathrm{A}$ similar rate of $70.6 \%$ was reported from previous Indian studies with natural streptokinase. ${ }^{[8]}$

Safety profile assessment which was the secondary end point in the present study was similar in both the r-SK and n-SK group. These events seen were similar to those reported by other studies. Incidence of bleeding was $4.2 \%$ (4) in the r-SK group while it was $5.4 \%$ (3) in the n-SK group, which are similar to the rates reported in literature vary between 0.5 to $15.2 \% .^{[1,5,11,13]}$ No anaphylactic reactions were seen in the r-SK group whereas one was reported from the n-SK group. Adverse effects of thrombolytic therapy commonly include hypotension $4.5-15 \%$, fever $5 \%$, rigors, nausea and vomiting $46 \%$ and bleeding (0.5-15.2\%). The mortality rates were low for both the groups $\{3.1 \%(3)$ and $5.5 \%(3)\}$, however the present small study did not have the statistical power to show the difference in the two comparable treatment groups. Post thrombolysis, cardiogenic shock was observed in $1(1.2 \%)$ and $2(3.6 \%)$ in the recombinant and natural streptokinase groups respectively whereas reinfarction was seen in $2(2.4 \%$ and $3.6 \%$ ) patients respectively in both the groups which is much less than in other studies.

The present study has its limitations as the sample size is small and the markers used for assessing reperfusion were non-invasive instead of the gold standard that is angiography, although the non-invasive methods have been validated against angiography and are proven surrogate markers of reperfusion. Since this trial was a randomised controlled trial the instigators were of the opinion that the conditions to see the efficacy and safety need some factors to be controlled including patients not more that 65 yers of age and without any co-orbid conditions that might influence the outcome. A larger study with patecny assessed by angiography and without influencing factors of age or others would give a precise assessment of reperfusion.

Streptokinase is the most widely used thrombolytic agent particularly in India while others like tPA are expensive. Reviews comparing other thrombolytic agents have shown that streptokinase to be as good as that of tPA or rPA in terms of both efficacy and safety. ${ }^{[14,15]}$ Recombinant streptokinase has the advantage of not containing streptolysin and streptodornase unlike streptococci-derived natural streptokinase which might make it safer, and will be cheap which is very relevant to our country's population. This technology can be further used to make more modifications in the present agents to make them more safe and efficacious. 


\section{REFERENCES}

1. Goa KL, Henwood JM, Langley MS, Clissold SP. Intravenous Streptokinase: A Reappraisal of its Therapeutic Use in Acute Myocardial Infarction. Drugs 1990;39:693-719.

2. AIMS trial study group. Effect of intravenous APSAC on mortality after acute myocardial infarction: Preliminary report of a placebo controlled clinical trial. Lancet 1988;1:545-9.

3. Simoons ML, Serruys PW, van den Brand M, Res J, Verheugt FW, Krauss XH, Remme WJ, et al. Early thrombolysis in acute myocardial infarction: Limitation of infarct size and improved survival. J Am Coll Cardiol 1986;7:717-28.

4. The GUSTO Investigators. An international randomized trial comparing four thrombolytic strategies for acute myocardial infarction. N Engl J Med 1993;329:673-82.

5. Gruppo Italiano per lo Studio della Soppravvivenza nell' Infart miocardico (GISSI2). A factorial randomized trial of alteplase vs. Streptokinase and heparin versus no heparin among 12490 patients with acute myocardial infarction. Lancet. 1992;339:753-70.

6. ISIS-3 (Third international Study of Infarct Survival). Collaborative Group. ISIS-3: A randomized comparison of streptokinase vs. tissue plasminogen activator vs. antistrplase and of aspirin plus heparin vs aspirin alone among 41299 cases of suspected acute myocardial infarction. Lancet 1992;339:753-70.

7. Christensen JH, Sorensen HT, Rasmussen SE, Ravn L, Nielsen FE. The effect of streptokinase on chest pain in acute myocardial infarction. Pain 1991;46:31-4.

8. Honloser SH, Zabel M, Kasper W, Thomas M, Just $\mathrm{H}$. Assessment of coronary artery patency after thrombolytic therapy; accurate prediction utilising the combined analysis of three noninvasive markers. J Am Col Cardiol 1991;18:44-9.

9. GISSI. Effectiveness of intravenous thrombolytics treatment in acute myocardial infarction. Lancet 1986;1:397-402.

10. The TREMA Group investigators. Multicenter, randomized, comparative study of recombinant vs. natural streptokinase in acute myocardial infarction. Thrombo Hemostat 1999;82:1605-9.

11. Streptokinase Multicentric trial group. Streptokinase in Acute Myocardial Infarction: A Multicentric Study in an Indian Setting. Ind Heart J 1993;45:189-94.

12. The TREMA Group investigators. TERMA-2 National extension of thrombolytic therapy with recombinant streptokinase in acute myocardial infarction. Thrombo Hemostat 2000;84:949-54.

13. GISSI. Long term affects of intravenous thrombolysis in acute myocardial infarction: Final report of GISSI study. Lancet 1987;2:871-4.

14. Boland A, Dundar Y, Bagust A, Haycox A, Hill R, Mujica MR, et al. Early thrombolysis for the treatment of acute myocardial infarction: A systematic review and economic evaluation. Health Technol Assess 2003;7:1-136.

15. Dundar Y, Hill R, Dickson R, Walley $T$. Comparative efficacy of thrombolytics in acute myocardial infarction: A systematic review. QJM. 2003;96:103-13. 